Background The NHS East of England guideline regarding administration of oxygen to infants (PNPG0161) was revised in 2019. It details the oxygen saturation target range and pulse oximetry monitoring alarm limits required by infants depending on their gestational age and comorbidities.

Objectives We set out to improve the prescribing and monitoring of oxygen saturation in the neonatal unit at Southend Hospital, with a view to making improvements should the standard fall below those set out in the guideline. Southend has a Level 2 unit which is able to care for infants born at from 27 weeks and monitors functional pulse oximetry using the Philips Intelliview MP70 Neonatal.

Methods We audited the unit between September and December 2019, 6 months after dissemination of the guideline within the neonatal department. This involved random spot checks of the prescriptions for oxygen and the saturation alarm limits set for all infants on the unit to assess compliance with the following 4 standards:

- $100 \%$ of infants receiving oxygen should have oxygen prescribed in a drug chart with a specified saturation target range.

- $100 \%$ of infants receiving oxygen should be on continuous pulse oximetry.

- $100 \%$ of continuous pulse oximetry should have appropriate set saturation alarm limits.

- $100 \%$ of infants requiring deviation from the recommendations should have this documented by a clinician in their notes.

Following the initial results, we worked with the unit manager and neonatal educational nurse to improve our performance. Departmental teaching was organised regarding the content of the guideline and we introduced a sticker into the oxygen section of the drug charts. This was to be acknowledged and validated twice daily by the nursing staff. We also displayed the recommended oxygen saturation targets for prescription and monitoring alarm limits on the unit and in the doctors' office.

Results 4 months after making improvements, we repeated random spot checks of the unit between May and June 2020. The overall rate of accurate oxygen prescribing rose from $22.9 \%$ to $87.9 \%$ and all cases with a correct prescription were associated with a sticker. The unit remained consistent in monitoring $100 \%$ of infants receiving oxygen, however, the rate of infants below 34 weeks gestation on continuous pulse oximetry fell to $95 \%$. The rate of correctly set saturation alarm limits rose from $81.9 \%$ to $85 \%$. Notably, it was term infants and those on air who frequently had incorrect settings. There remained no cases in which deviation from the guidelines was justified in the notes.

Conclusions Certain improvements, particularly use of a sticker in the drug charts, increased the accuracy with which oxygen saturation target ranges were prescribed. Further improvements are required for our unit to achieve the standards set by NHS East of England, such as ensuring all infants have an oxygen prescription and correctly set saturation alarm limits. We recommend augmentation of the sticker with more information and space to document justification of any deviation from the guideline. We suggest further departmental teaching regarding this, in particular the pulse oximetry monitoring of term infants on air and those younger than 34 weeks.
British Association for Paediatric Nephrology

\section{A SYSTEMATIC REVIEW OF URINE BIOMARKERS IN CHILDREN WITH IGA VASCULITIS NEPHRITIS}

${ }^{1}$ Chloe Williams, ${ }^{2}$ Aileen Toner, ${ }^{3}$ Rachael Wright, ${ }^{4}$ Louise Oni. ${ }^{1}$ School of Medicine, University of Liverpool, Liverpool, UKDepartment of Women's and Children's Health, Institute of Life Course and Medical Sciences, University of Liverpool, Liverpool, UK; ${ }^{2}$ School of Medicine, University of Liverpool, Liverpool, UK; ${ }^{3}$ Department of Women's and Children's Health, Institute of Life Course and Medical Sciences, University of Liverpool, Liverpool, UKi ${ }^{4}$ Department of Paediatric Nephrology, Alder Hey Children's NHS Foundation Trust Hospital, Liverpool, UK

\subsection{6/archdischild-2021-rcpch.61}

Background Nephritis is a recognised complication of IgA vasculitis (IgAV, Henoch-Schoenlein purpura, HSP) and contributes to $1-2 \%$ of all chronic kidney disease (CKD). Improved detection and understanding of kidney inflammation may reduce irreversible kidney damage in IgA vasculitis nephritis (IgAV-N).

Objectives The primary aim of this study was to perform a comprehensive systematic review to evaluate the current literature to identify promising urine biomarkers that can predict and assess the severity of kidney disease in children with IgAV.

Methods A systematic literature review was performed using 4 search engines and a search term strategy with predefined inclusion and exclusion criteria. Promising biomarkers were divided in terms of clinical or pre-clinical and described using statistical significance and area under the curve (AUC) values.

Results 121 studies were identified and thirteen were eligible for inclusion. A total of 2,446 paediatric patients were included; 51\% male, median age 7.9 years (range not available). This comprised of healthy controls $(n=761)$, children with IgAV-N $(n=1,236)$ and children with IgAV without nephritis (IgAV-noN, $n=449$ ). The clinical markers for assessing severity of nephritis, 24-hour protein quantity, urine protein: creatinine ratio were both acceptable (AUC <0.8) and urinary albumin concentration (Malb) performed well (AUC 0.810.98). The most promising pre-clinical urinary biomarkers were kidney injury molecule-1 (KIM-1) (AUC 0.93), monocyte chemotactic protein-1 (MCP-1) (AUC 0.83), N-acetyl-beta-glucosaminidase (NAG) (0.76-0.96), and angiotensinogen (AGT) (no AUC available).

Conclusions Further longitudinal studies are needed to assess whether these biomarkers enhance standard of care in the management of IgAV-N.

\section{Paediatricians with Expertise in Cardiology Special Interest Group}

\section{MAKING PAEDIATRIC ECG INTERPRETATION IN THE PAEDIATRIC EMERGENCYDEPARTMENT EASIER AND SAFER BY INTRODUCTION OF AN ECG CHECKLIST}

${ }^{1}$ Jamie Wood, ${ }^{2}$ Karen McLeod, ${ }^{2}$ Steven Foster. ${ }^{1}$ NHS Forth Valley; ${ }^{2}$ NHS Greater Glasgow and Clyde

10.1136/archdischild-2021-repch.62 
Background The accuracy of reporting electrocardiograms by trainees in paediatric emergency medicine has been shown to increase with experience. However, most paediatric trainees will only spend 3-6 months in the emergency department with limited opportunity to improve skills in electrocardiogram reporting.

Interpretation in the emergency department has been shown to be relatively inaccurate and additional reporting of emergency department electrocardiograms by a consultant paediatric cardiologist increases the diagnostic accuracy. As a result, in many paediatric cardiac units the burden of electrocardiogram reporting is placed on the cardiology team, resulting in a significant workload. In addition, time taken for electrocardiograms to be reviewed by reporting teams may result in delay to clinic referral for patients with electrocardiogram abnormality.

A previous study has shown that even amongst paediatricians, accuracy at interpreting paediatric ECGs is only around $60 \%$. Although, there are accepted normal ranges and values for paediatric electrocardiograms, these are often presented in busy tables that can be complex and daunting to use, especially in a time pressured clinical environment.

We hypothesised that a diagnostic aid, in the form of an electrocardiogram checklist, could assist in electrocardiogram interpretation, helping to screen for electrocardiograms that needed to be reviewed by a cardiologist and reducing the time to cardiology review for patients with electrocardiogram abnormalities.

Objectives We set out to assess the use of a simple checklist and guideline to aid interpretation of paediatric electrocardiograms in the paediatric emergency department.

Methods An electrocardiogram interpretation checklist and guideline were implemented in the emergency department. Abnormal electrocardiograms identified by the checklist were reviewed by a paediatric cardiologist and patients appointed to a cardiology outpatient clinic. The process was prospectively evaluated over six months to determine the ability of the checklist to detect abnormal electrocardiograms. The emergency department clinicians were sent a questionnaire to evaluate their experience with the checklist.

Results Between May and November 2018, 600 electrocardiograms were performed in paediatric emergency department. 48 electrocardiograms of patients known to cardiology services or discussed with the on-call team were excluded. Of the remaining 552 electrocardiograms, 30 were identified by the emergency clinicians as abnormal and sent for cardiology review. 13/30 of these were considered normal by the consultant cardiologist and the patients discharged. The other 17 patients were allocated to cardiology outpatient clinic. Only 3/ 17 required ongoing follow-up. Of the 522 electrocardiograms deemed normal by the emergency department clinicians, cardiology disagreed in $8(1.4 \%)$. In these cases, there was either incorrect lead placement or the checklist had been applied incorrectly. All 8 patients were seen in cardiology outpatient clinic but subsequently discharged. Use of the checklist demonstrated an excellent negative predictive value of 98.47\% [CI $97.32 \%$ to $99.13 \%]$. Following implementation, time from emergency department attendance to outpatient clinic decreased from a median of 89 to 45 days $(\mathrm{P}<0.001)$ and survey respondents reported increased confidence in interpreting paediatric electrocardiograms.

Conclusions The use of a simple checklist and guideline allows confident and accurate detection of electrocardiogram abnormality by emergency department staff and speeds referral to cardiology clinic for patients with electrocardiogram abnormalities.

\section{British Paediatric Allergy Immunity and Infection Group}

\section{AUDIT OF SPECIFIC IGE TESTING IN PAEDIATRICS DEPARTMENT SLIGO UNIVERSITY HOSPITAL, SLIGO}

${ }^{1}$ Asad Nasim, ${ }^{2}$ Tummaluru Rohininath. 'Wexham Park Hospital, Slough; ${ }^{2}$ Sligo University Hospital, Sligo, Ireland

\subsection{6/archdischild-2021-rcpch.63}

Background 5-6\% children suffer with food allergy in Republic of Ireland.

In UK 3-6\% preschool and 1-2\% of older children suffer with food allergy.

Most common food allergens are milk, egg, nuts, fish.

Most children present with atopic conditions.

$38.7 \%$ of these children present with severe allergic reaction/anaphylactic reaction which is life threatening. These children require emergency management including adrenaline.

In children with food allergies,38.4\% are allergic to multiple foods.

Objectives To assess the pattern of results of specific $\operatorname{IgE}$ Testing.

To see Indications of Epipen prescription for children with allergic reactions being met or not.

Methods Retrospective review of the charts who got specific IgE Testing in Sligo University Hospital. These patients were referred through Paediatrics, ENT OPD or GP between July 2015 to January 2016.

A proforma was made to take the data and it contained the names of the patient with coding, age, associated family and personal history of allergies/asthma/atopic eczema, indication for the testing, results of the test, whether prescribed Epipen or not.

Completed data was analysed and report was generated.

Results $45 \%$ pts were < 3 yrs old who were tested for Specific IgE levels.

$13.3 \%$ patients included in the audit were meeting the criteria to prescribe Epipen.

$50 \%$ of the total 8 pts meeting the guidelines were not prescribed Epipen.

Total IgE levels can be normal, as in this audit $61.7 \%$ pts had normal IgE level. These can be falsely high as in 14 out of 22 patients with high IgE levels there was no history of anaphylaxis or severe allergic reaction.

Nuts(27\%), $\operatorname{Egg}(21 \%)$ and milk(8\%) allergy are commonest among food allergies along with grass and house dust mite common as co allergens.

Personal history of asthma was +ve in $16.6 \%$ patients and for atopic eczema was +ve in $26.6 \%$ patients.

Family history of asthma was +ve in $10 \%$, for atopic eczema in $6.6 \%$ and for food allergies it was +ve for $33.3 \%$ patients.

Conclusions Allergy focused history should be taken and Specific IgE testing should only be done when indicated in the history and for the suspected allergens. 